\title{
Inventari consonantici dell'italiano e del polacco a confronto
}

\author{
The comparison of phonological inventories of Italian \\ and Polish consonants \\ Olga BRONIŚ ${ }^{1}$ \\ Uniwersytet Kardynała Stefana Wyszyńskiego w Warszawie
}

\begin{abstract}
The goal of the present paper is to offer a comparative study of the consonantal phonological inventories of Italian and Polish. The first discrepancy between the two languages discussed in the paper is related to the fact that some Polish and Italian phonemes do not have any correspondents in the other language. The second issue addressed in this work is the fact that some Italian segments seem very similar to selected Polish sounds, and yet, as the paper shows, in some cases substantial differences need to be noted. The last issue presented in the paper concerns selected properties of Italian and Polish geminates.
\end{abstract}

Keywords: phonetics, phonology, pronunciation, Italian, Polish, contrastive grammar

\begin{abstract}
Lo scopo di questo lavoro è quello di effettuare uno studio comparativo degli inventari consonantici dell'italiano e del polacco. La prima differenza tra le due lingue di cui parla il presente saggio è legata all'assenza dei fonemi corrispondenti in uno dei due sistemi. La seconda questione sollevata nel lavoro è l'apparente somiglianza di alcune consonanti italiane e polacche, che, pur essendo simili nell'italiano e nel polacco, presentano discrepanze fonetiche e fonologiche molto significative. L'ultimo argomento compreso nell'analisi è il confronto delle geminate dell'italiano e del polacco.
\end{abstract}

Keywords: fonetica, fonologia, pronuncia, italiano, polacco, grammatica contrastiva

1 (D) https://orcid.org/0000-0002-5323-0665. 


\section{Introduzione}

Nell'articolo del 2010 Stephen R. Anderson cerca di stabilire quante lingue ci siano nel mondo. Basandosi sulle informazioni estratte dal catalogo Ethnologue, pubblicato online dalla SIL International (Summer Institute of Linguistics), Anderson riporta che oggigiorno sul nostro pianeta esistono ca. 7000 lingue. Data la stima e il prestigio che gode il catalogo Ethnologue, non ci sono dubbi sul fatto che le lingue riportate in questo documento siano dei sistemi autonomi e variegati. Inoltre, Anderson osserva che il numero di lingue nel mondo potrebbe essere ancora più grande se si cambiassero un po' i parametri che formano i criteri distintivi di questa classificazione. Di sicuro, uno dei parametri di base che distinguono un sistema linguistico dall'altro è il numero e il repertorio dei fonemi usati dai parlanti di questa lingua. L'insieme dei fonemi che formano l'ossatura del sistema fonologico di una lingua è detto inventario fonologico. Ogni lingua ha a propria disposizione un inventario di fonemi, ovvero dei suoni linguistici, detti anche foni, che in questa lingua hanno una funzione distintiva, come in italiano, per esempio, /1/ e /n/, che dimostrano di essere la base della coppia minima (Trubetzkoy, 1939) solo sono.

La situazione linguistica in Polonia è molto diversa da quella in Italia. Prima di tutto, in Polonia non esistono molti dialetti e lingue regionali che, a loro volta, vengono usati da relativamente pochi parlanti ${ }^{2}$. In Italia, invece, i dialetti sono numerosi. Secondo Anderson (2010), se si applicassero dei criteri più larghi nella definizione di una lingua, solo nell'Italia settentrionale ne risulterebbero circa 500. Accanto a questi dialetti, esiste in Italia la lingua standard, parlata e utilizzata in tutto il paese, detta, appunto, italiano standard. A questo proposito, perciò, viene spesso contestato che parecchi italiani siano bilingui (Dardano e Trifone, 1995/2016, p. 44-45). Anche in Polonia esiste il cosiddetto polacco standard, che, comunque, a differenza dell'italiano, di solito costituisce l'unica lingua L1 acquisita dai polacchi.

Lo scopo di questo lavoro è quello di presentare l'inventario delle consonanti fonemiche della lingua italiana e polacca, e poi, confrontare i due sistemi. All'analisi sincronica presentata nell'articolo saranno sottoposte le moderne lingue standard, quella italiana e polacca. Il lavoro si basa sulle tradizionali classifiche articolatorie delle consonanti, create e aggiornate da numerosi ricercatori, riportate e considerate nelle sezioni successive.

2 Per informazioni sui dialetti polacchi, vide il compendio dei dialetti polacchi della Karaś http://www.dialektologia.uw.edu.pl/index.php. 


\section{Inventario delle consonanti italiane}

Tradizionalmente, le consonanti si distinguono in categorie che si basano sulle caratteristiche articolatorie, relative alla produzione di un suono linguistico. Oggi le consonanti si possono definire anche con termini legati alla fonetica acustica. Tuttavia, visto che l'utilizzo della terminologia acustica richiede una certa conoscenza della fisica acustica, di solito si continuano a classificare i fonemi consonantici con l'uso dei criteri articolatori. Altresì, la descrizione acustica mostra le caratteristiche di una specifica e individuale realizzazione di un fono, mentre analizzando l'astratto sistema fonologico della lingua, esso sembra meglio fare uso dei criteri ben determinati, nonostante siano astratti, e che costituiscono un certo punto di riferimento per la classificazione di ogni fono prodotto in una lingua. Questo scopo può essere ben ottenuto con la classificazione articolatoria, menzionata sopra. Dal punto di vista della fonetica articolatoria, le consonanti si definiscono con l'ausilio di cinque criteri principali:

(1) Criteri di classificazione delle consonanti con il riferimento alla fonetica articolatoria:

a) l'uso dell'aria nella fase espirativa o no: polmonare - non-polmonare

b) la posizione delle corde vocali: sordo - sonoro

c) la posizione del velo palatino: nasale - orale

d) il luogo di articolazione

e) il modo di articolazione

Il primo criterio (1a) non è molto utile per il confronto delle consonanti polacche e quelle italiane. Dato che il processo di articolazione di ogni consonante, sia polacca che italiana, comincia dall'uscita dell'aria dai polmoni, se ne deduce che tutte le consonanti italiane e polacche, come le consonanti della maggior parte delle lingue del mondo, siano di tipo espirativo. Il criterio (1b), che distingue le consonanti sonore da quelle sorde, è invece valido per la classificazione delle consonanti in tutte e due le lingue. In esse, infatti, le consonanti possono essere sorde o sonore. Tuttavia, nonostante il criterio (1b) sia indispensabile per la classificazione delle consonanti italiane e polacche, esso non è rilevante per la presente discussione che ha come scopo il confronto dei due inventari fonologici. Sarà utile nella descrizione dei foni, ma non è una caratteristica distintiva, come tale. Lo stesso si può dire a proposito del criterio (1c). Le consonanti sia italiane che polacche possono essere orali o nasali. Questa distinzione, comunque, non è rilevante se si vogliono contrastare i due 
inventari, ma di sicuro ci aiuterà nella descrizione dei foni analizzati. Infatti, i criteri che costituiscono i fondamenti della discussione comparativa tra i sistemi consonantici dell'italiano e del polacco sono gli ultimi due, ovvero il luogo (1d) e il modo (1e) di articolazione. A questo punto, consideriamo la tabella riportata qui a seguito, che raccoglie tutti i fonemi italiani e polacchi, sistemati secondo i due criteri (1d) e (1e).

(1) Le consonanti fonemiche italiane e polacche

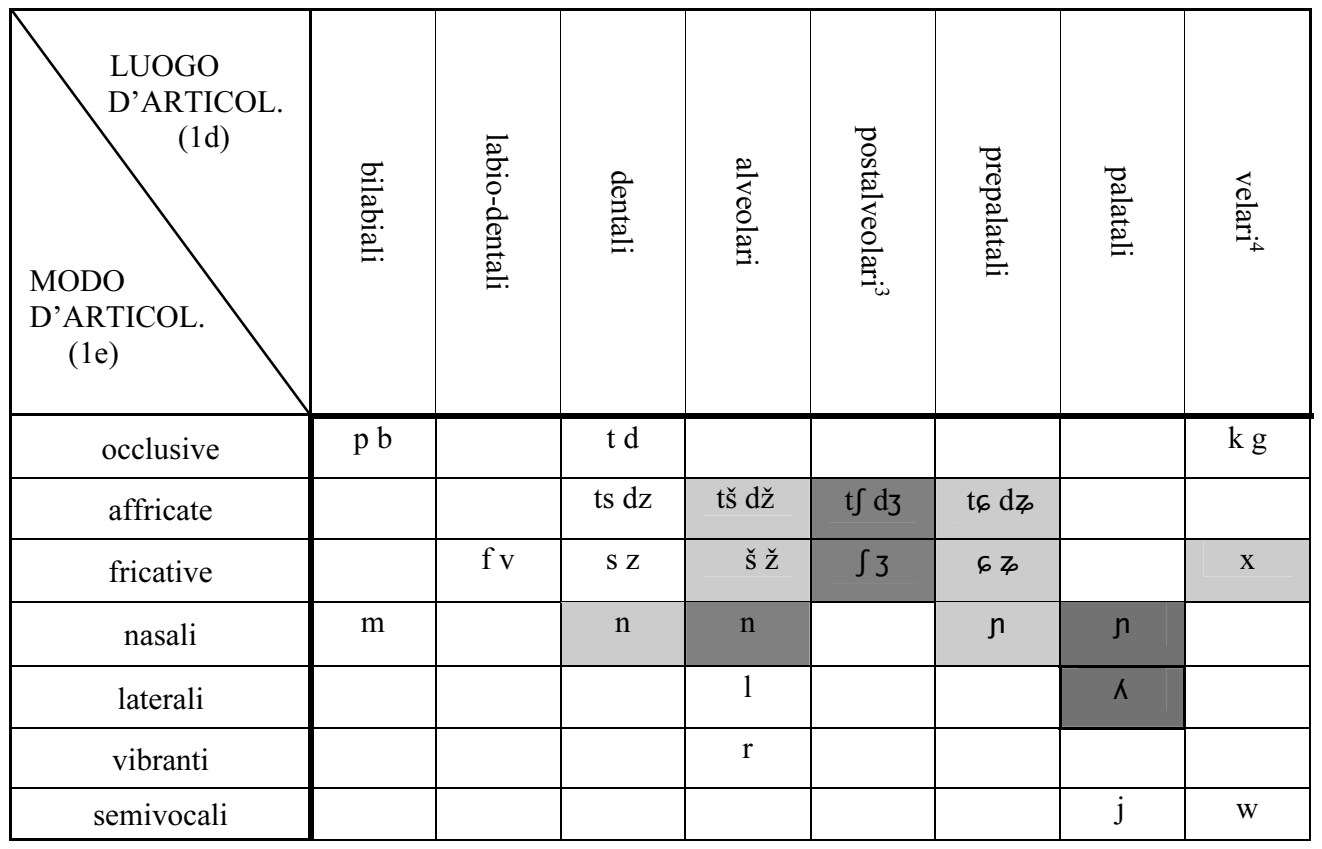

Nella tabella le colonne raffigurano i vari luoghi di articolazione delle consonanti, e le righe le organizzano a seconda del modo in cui avviene la loro articolazione. I simboli nelle celle bianche rappresentano i fonemi presenti sia in italiano che in polacco, mentre gli altri simboli, con la bandiera polacca o italiana sullo sfondo, raffigurano i fonemi presenti in una sola delle due lingue. La tabella è stata preparata sulla base dei lavori della Wierzchowska

3 Postalveolari secondo Bertinetto e Loporcaro (2005), postalveopalato-prolabianti secondo Canepari (2006).

${ }^{4}$ Nella classificazione di Bertinetto e Loporcaro (2005), la semivocale /w/ è definita come labialevelare. 
(1971, 1980), Rubach $(1977,1984)$ per il polacco5 , e di Bertinetto e Loporcaro (2005) e Canepari (2006) per l'italiano'.

Nella tabella si presentano tutte le consonanti fonemiche dell'italiano e del polacco. Le consonanti sono codificate con i simboli dell'IPA (International Phonetic Alphabet). Solo due consonanti polacche non hanno un simbolo rappresentativo nell'IPA, perciò, a seguito di Rubach (1977, 1984, inter alia), nella presente classifica, vengono utilizzati due simboli provenienti dalla tradizione slava: /š/ e /ž/. La necessità di introdurre i simboli fuori-IPA è legata al fatto che le consonanti polacche /š/ e /ž/ sono dal punto di vista della fonetica sia articolatoria che acustica diverse dalle consonanti / $/ \mathrm{s} / \mathrm{e} / 3 /$, presenti, tra l'altro, in italiano. Si potrebbe mettere in discussione la scelta dei simboli che rappresentano i foni mancanti dalla tabella dell'IPA. Il presente lavoro accetta i simboli /š/ e /ž/, provenienti del SPA (Slavic Phonetic Alphabet) invece di usare dei segni arbitrari, come lo fa, per esempio, la Kwapisz-Osadnik $(2012)^{7}$.

Lo studio di altri lavori dedicati alla fonetica italiana rivela alcune discrepanze rispetto alla classificazione proposta da Bertinetto e Loporcaro (2005), su cui si basa la tabella in (2). Per esempio, Albano Leoni e Maturi (1998) e Maturi (2006) ritengono che le consonanti /t d s z t dz/ siano alveolari, non dentali, come ritengono Bertinetto e Loporcaro. Canepari (2006) ritiene invece che gli stessi fonemi siano dentali. Discrepanze del genere si osservano anche in altri manuali di fonetica. Per esempio, nella classificazione di D'Achille (2010) /t d n/ appartengono al gruppo dei fonemi dentali, mentre /s $\mathrm{z}$ ts $\mathrm{dz} /$ sono considerati alveolari, come /l/ e /r/. Un'altra differenza tra la classificazione di Bertinetto e Loporcaro (2005) e quella di D'Achille riguarda la nominazione del luogo di articolazione delle consonanti $/ \int \quad 3 \mathrm{t} \int \mathrm{d} /$. A prescindere dal fatto che nella classificazione di D'Achille manca il fono /3/,

\footnotetext{
${ }^{5}$ La stessa classificazione dei luoghi e modi di articolazione delle consonanti polacche è citata nei lavori di Wiśniewski (2001) e dell'Ostaszewska e della Tambor (2000).

${ }^{6}$ Non tutti i ricercatori classificano le consonanti italiane secondo gli stessi criteri. Le discrepanze tra la classificazione presentata in (2) e quelle introdotte da altri linguisti saranno analizzate nella parte successiva della presente sezione.

$7 \mathrm{Va}$ notato che il presente saggio segue la notazione dell'IPA anche per quanto riguarda la trascrizione delle affricate, evidenziando allo stesso tempo il fatto che siano composte da due foni diversi. Così, nella tabella in (2) sono incluse le affricate /ts/, /ds/, /tš/, /dž/, /tts/, /dz/, /tø̌/, $/ \mathrm{d} \iota_{/}$, tutte trascritte come la sequenza di due foni diversi. Al contrario, il SPA rinuncia di mostrate la duplice natura dell'affricata, trascrivendo, per esempio, /dž/ come /ふ̌/ o /tš/ come /č/. Nonostante ciò, i ricercatori polacchi che usano il sistema SPA, come Wiśniewski (2001) e l'Ostaszewska e la Tambor (2000), seguono gli indici del sistema anche in proposito alla trascrizione delle affricate.
} 
lo stesso ricercatore definisce il luogo di articolazione dei rimanenti foni postalveolari $/ \int \mathrm{t} \int \mathrm{d} 3 /$ come palatale, che è piuttosto distante dal luogo di articolazione presentato nella tabella in (2), ovvero postalveolare. Invece, Maturi (2006) ritiene che le consonanti $/ \int 3 \mathrm{t} \int \mathrm{d} 3 /$ siano prepalatali, non postalveolari, mentre Albano Leoni e Maturi (1998) ritengono che i due termini, prepalatale e postalveolare, siano sinonimi. Infine, secondo Canepari (2006) i fonemi $/ \int 3 \mathrm{t} \int \mathrm{d} 3 /$ appartengono al gruppo detto postalveopalatoprolabiali.

In sintesi, sembra che le incongruenze che emergono da alcune fonti siano limitate a due luoghi di articolazione: dentale e alveolare come il primo luogo d'inconsistenza, e postalveolare-(pre)palatale come il secondo. A questo punto si deve osservare che sia il numero dei foni italiani che il modo di classificarli è disputabile e dipende dai presupposti teorici e dal livello d'astrazione considerata accettabile dal punto di vista dell'analisi. Al momento, la classificazione che viene considerata esemplare per l'italiano è quella coniata da Bertinetto e Loporcaro (2005), la cui esattezza permette di catturare le sottili sfumatureche distinguono in modo adeguato le consonanti italiane da quelle polacche. Ovviamente, si possono adottare altri sistemi classificativi, come quello di Mioni (1993), citato ed effettivamente accolto da Krämer (2009). Nella classifica di Mioni (1993) è fortemente ridotto il numero dei luoghi di articolazione usati nella descrizione dell'italiano a solo quelli che sono contrastivi dal punto di vista fonologico. Questoapproccio riduttivo è molto utile nell'analisi generativa della fonologia, che si basa, tra l'altro, sul concetto dell'economia. Al contrario dell'analisi generativa dei processi fonologici di Krämer, il presente lavoro si concentra sulla discussione contrastiva che riguarda la rappresentazione segmentale dei fonemi italiani e polacchi. Qui l'astratto non è gradito, perché l'analisi contrastiva degli inventari consonantici deve evidenziare le differenze articolatorie tra $\mathrm{i}$ foni delle due lingue; Perciò la classificazione adottata nell'analisi è quella di Bertinetto e Loporcaro (2005).

Per quanto riguarda la classificazione dei fonemi consonantici del polacco, nella bibliografia sull'argomento non si notano dei punti incongruenti, che, al contrario, si osservano ampiamente nel caso dell'italiano. Nella descrizione del polacco quasi tutti i ricercatori, inclusi quelli citati nel presente lavoro, seguono la Wierzchowska (1971, 1980), pioniere nel campo della fonetica polacca che ha gettato le fondamenta per la discussione fonetica e fonologica sulla lingua polacca. Anche gli autori come l'Ostaszewska 
e la Tambor (2000), oppure Wiśniewski (2001) utilizzano il raggruppamento della Wierzchowska ${ }^{8}$.

Per quanto riguarda l'analisi contrastiva dei fonemi consonantici italiani e polacchi, dalla tabella emergono due questioni principali: l'assenza dei suoni corrispondenti nelle due lingue e la diversità nei luoghi di articolazione delle consonanti italiane e polacche che molto spesso sono interpretate come foni molto simili o, anzi, uguali. I due argomenti saranno approfonditi nelle sezioni 3 e 4 , rispettivamente. Successivamente, nella parte 5 saranno presentate a confronto le consonanti lunghe polacche e italiane.

\section{Assenza dei fonemi corrispondenti}

Considerando la tabella in (2), notiamo che ci sono dei fonemi tipici di solo una delle due lingue. Diversamente dai fonemi come la / $t /$, che esiste in tutte e due le lingue, nella tabella si osservano delle consonanti che sembrano isolate, non accoppiate. Si tratta soprattutto del fonema polacco $/ \mathrm{x} /{ }^{1}$ e di quello italiano $/ N^{10}$. Per quanto riguarda il fonema italiano $/ N /$, nel polacco non è presente nessun suono simile. In tutte e due le lingue c'è un'altra consonante laterale, la / $1 /$, che in un certo modo aiuta a comprendere e a spiegare il modo di articolazione della laterale $/ \mathrm{N}$. Tuttavia, questi due foni sono molto diversi a causa della distanza dei loro luoghi di articolazione. Nella pronuncia di /// viene attivata la punta della lingua, mentre l'articolazione di / $/$ richiede l'uso del dorso della lingua che si avvicina al palato, e non, come succede nel caso della /1/, alla cresta alveolare.

8 Come segnalato sopra, anche la classificazione delle consonanti polacche riportata nel presente lavoro si basa sugli studi della Wierzchowska. Va detto, comunque, che il raggruppamento scelto dall'autrice non include le consonanti dolci, ossia ammorbidite, che lo rende diverso dalle classifiche standard dei linguisti polacchi. Il motivo per l'esclusione delle consonanti dolci dalla tabella sono spiegati nella sezione 3 .

${ }^{9} \mathrm{Nel}$ libro della Kwapisz-Osadnik (2012) la fricativa velare sorda / $\mathrm{x} /$ è raffigurata dal simbolo /h/, che nella nomenclatura dell'IPA rappresenta un suono assai diverso, ovvero la fricativa glottale sorda presente, per esempio, all'inizio della parola inglese house.

${ }^{10}$ In un certo modo, anche $\mathrm{i}$ fonemi $/ 6$ दo t6 $\mathrm{d}$ / $/$ possono essere interpretati come privi di corrispondenti, perché i loro potenziali compagni, le consonanti italiane $/ \int 3 \mathrm{t} \int \mathrm{d} /$, di solito vengono confuse e collegate con le consonanti alveolari polacche /š ž tš dž/. Comunque, visto che

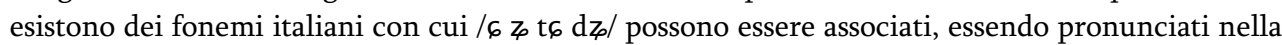
stessa maniera ma da due punti di articolazione diversi, si ritiene che queste quattro consonanti non siano uniche ed incomparabili come i due foni $/ \mathrm{x} / \mathrm{e} / \mathrm{N}$. A questo proposito, dunque, i foni / 6 ६ t $6 \mathrm{~d}$ / / saranno esaminati nella sezione successiva, dedicata ai fonemi con dei corrispondenti aventi simili luoghi di articolazione. 
L'altro fonema che esiste in uno solo dei due sistemi esaminati è la fricativa velare sorda polacca $/ x /$. Questo fonema è rappresentato nel polacco dalla lettera $h$, che in italiano è detta muta e non presenta nessun corrispondente nella pronuncia. La pronuncia del fono $/ \mathrm{x} /$ risulta piuttosto difficile per la maggior parte degli italiani. Dato che $/ x /$ è rappresentato nell'ortografia dalla lettera $h$, o come $c h^{11}$, il fono viene spesso omesso oppure realizzato come $/ \mathrm{k} /$.

Un altro argomento che deve essere sollevato nella discussione sui fonemi senza corrispondenti in una delle due lingue analizzate è il contrasto tra le consonanti dure e morbide. Nel libro dell'Ostaszewska e della Tambor (2000), come in Wiśniewski (2001), la classificazione delle consonanti polacche include due varianti delle consonanti non-palatali: uno duro e l'altro morbido (detto anche dolce), dove la dolcezza della consonante è rappresentata dal segno diacritico $/ \%$. In seguito ai lavori fonetici della Wierzchowska (1971), sia Wiśniewski (2001) che l'Ostaszewska e la Tambor (2000) distinguono le consonanti dure, intrinsecamente morbide (palatali) e ammorbidite (dolci), dove la morbidezza e l'effetto dell'attivazione di un secondario luogo di articolazione ${ }^{12}$. Il motivo di includere nelle classificazioni delle consonanti polacche dell'Ostaszewska e della Tambor (2000) e di Wisniewski (2001) non solo le varianti dure delle consonanti non-palatali ma anche quelle ammorbidite, è probabilmente causato dal fatto che la Wierzchowska, nei suoi testi canonici sulla fonetica $(1971,1980)$, offre una descrizione molto ampia sia delle consonanti dure che ammorbidite. Dato che in polacco le consonanti dolci si osservano a largo, non sorprende il fatto che la Wierzchowska le abbia dedicato molto spazio nel suo lavoro. Va detto, comunque, che la ricerca della Wierzchowska venne condotta ai tempi in cui la fonologia come una disciplina indipendente era ancora agli arbori, perciò i lavori della stessa Wierzchowska hanno soprattutto un carattere fonetico. Non ci sono dubbi che a livello fonetico, sia acustico che articolatorio, le consonanti dure sono molto diverse da

\footnotetext{
${ }^{11}$ In alcuni varianti regionali del polacco e nelle sue forme arcaiche esiste non solo la fricativa velare sorda $/ \mathrm{x} /$, ma anche il suo corrispondente sonoro $/ \gamma /$. Tuttavia, dato il carattere sincronico del presente lavoro, questo argomento non farà parte dell'analisi.

12 Dal punto di vista del processo articolatorio, è risaputo che le consonanti dolci, ossia ammorbidite, sono i foni prodotti con un addizionale gesto articolatorio, che oltre ad aver un ben preciso luogo di articolazione, sono realizzate tramite l'alzamento della parte centrale della lingua verso il palato duro. La Wierzchowska (1971) nota anche che la differenza nell'articolazione delle consonanti dure e morbide in genere non è legata solo alla posizione orizzontale della lingua, ma pure alla forma generale del canale articolatorio, che nella produzione delle consonanti morbide assomiglia molto alla forma del canale tipico della produzione della vocale /i/.
} 
quelle morbide. Nondimeno, va detto che l'inventario fonemico delle consonanti di una lingua non deve necessariamente includere ogni fono presente in questa lingua. Al contrario, ci deve essere una forte motivazione fonologica per includere un fono nella classifica dei fonemi. Inoltre, il sistema consonantico dovrebbe essere compatibile con quello vocalico.

In seguito a Rubach (1984, 2018, inter alia), il presente lavoro ritiene che la vocale $/ \mathfrak{t} /$ abbia lo status di un fonema ${ }^{13}$. Nell'ipotesi che $/ \mathfrak{t} /$ sia un fonema, tutte le consonanti dolci, cioè non morbide intrinsecamente, diventano ammorbidite sotto l'influenza della vocale anteriore $/ \mathrm{i} / \mathrm{o} / \varepsilon /$, oppure della semivocale anteriore /j/, tramite il processo di palatalizzazione (Rubach 1984, 2018). Dato il prerequisito sullo status fonologico della vocale $\mathrm{di} / \mathrm{t} /$, le consonanti dolci nel polacco non svolgono la funzione dei fonemi, ma piuttosto degli allofoni. Ergo, non appartengono all'inventario consonantico del polacco. Ecco il motivo per cui le consonanti ammorbidite non sono incluse nella tabella (2).

\section{Il luogo di articolazione}

Riguardo allo studio comparativo dell'inventario fonologico dell'italiano e del polacco, in entrambe le lingue esistono delle consonanti che da alcuni sono percepite come identiche, anche se in realtà non lo sono ${ }^{14}$. Per fare un esempio, consideriamo una coppia di falsi amici italo-polacchi. La parola italiana croce /'krotse/15 e la parola polacca krocze/'krotš̌/ "(io) cammino" (Broniś, 2017) sono spesso interpretate come simili, o addirittura uguali ${ }^{16}$. Invece, come risulta dalla

\footnotetext{
${ }^{13}$ Per la discussione fonologica sullo status fonologico della / $\mathbf{t} /$, si faccia riferimento a Rydzewski (2016).

14 Per esempio, la Kwapisz-Osadnik (2012) ritiene che le consonanti $/ \int \mathrm{t} \int \mathrm{d} 3 /$ facciano parte dell'inventario sia polacco che italiano, essendo in entrambe le lingue prepalatali.

15 La trascrizione delle parole italiane è effettuata sulla base del dizionario di Canepari (1999/2009). Per quanto riguarda il polacco, tutte le pronunce riportate nell'articolo si basano sul sistema di trascrizione di IPA, adattato da Rubach (1977,1984, inter alia). In linea con l'approccio di Canepari (1999/2009), la lunghezza vocalica non è contenuta nella trascrizione fonemica, presentata nel presente articolo.

${ }^{16}$ Secondo la classificazione della Kwapisz-Osadnik (2012) non solo le fricative e affricate /s z ts $\mathrm{dz} /$ hanno in polacco e in italiano un identico luogo di articolazione. L'autrice considera identiche anche le postalveolari fricative e affricate italiane $/ \int \mathrm{t} \int \mathrm{d} 3 /$ e quelle alveolari polacche $/ \check{s}$ tš dž/, descrivendo tutti questi foni ugualmente con i simboli dell'IPA per le due lingue. A questo punto vale la pena di aggiungere che secondo la Kwapisz-Osadnik /3/ non è un fonema italiano. Quest'atteggiamento, per esempio, non è diviso da Canepari (1999/2009) il quale nel suo
} 
trascrizione fonetica delle due parole, la loro pronuncia non è identica. Secondo le informazioni riportate nella tabella (2), la consonante italiana / $\mathrm{t} / \mathrm{e}$ la polacca /tšs sono tutte e due le affricate che si realizzano in punti di articolazione molto simili, ma non uguali. Sia /t $\mathrm{s} /$ sia/tš/ si producono nella zona della cresta alveolare, ma il proprio punto di articolazione di questi foni non è pienamente identico. Quello che garantisce la realizzazione corretta del fonema polacco è il posizionamento più anteriore della lingua rispetto a quello del fono italiano, e perciò le affricate e fricative $/ s \quad 3$ t $s \mathrm{~d} / /$ italiane sono definite come postalveolari, mentre quelle polacche, ossia /š ž tš dž/, come alveolari.

Un'altra differenza articolatoria tra le fricative e affricate alveolari polacche e le consonanti postalveolari italiane è relativa all'arrotondamento della bocca (Canepari 2006) che è un elemento considerato indispensabile dal punto di vista dell'articolazione dei foni italiani. Nel caso delle fricative e affricate alveolari polacche, l'arrotondamento della bocca non viene considerato rilevante.

Come mostrato sopra, le consonanti postalveolari italiane $/ \int 3 \mathrm{t} \int \mathrm{d} z /$ sono confrontabili con le consonanti alveolari polacche /š ž tš dž/. Comunque, le fricative e le affricate postalveolari dell'italiano potrebbero anche essere confrontate con un altro gruppo di fricative e affricate polacche, il cui luogo di articolazione è ancora più posteriore di quello delle consonanti italiane $/ \int 3 \mathrm{t} \int \mathrm{d} 3 /$. Si tratta dei fonemi prepalatali polacchi /6 $4 \mathrm{t} 6 \mathrm{~d} \% /$.

Tutti i polacchi riconoscono le fricative e affricate alveolari /š ž tš dž/

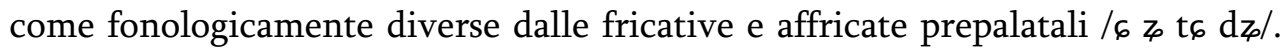
Lo dimostrano numerosi esempi di coppie minime, come Kasia ['kąa] 'Caterinina (nome fem., forma diminutiva)' e kasza ['kaša] 'grano'. Intuitivamente, i polacchi sanno dove posizionare e come modellare la fronte della lingua per ottenere il fono desiderato, anche se dal punto di vista dell'articolazione la differenza tra i due gruppi di foni è minima. Tornando al punto: come mostra la tabella (2), il luogo di articolazione delle consonanti $/ \int 3 \mathrm{t} \int \mathrm{d} 3 /$ si trova a metà strada tra il posto di articolazione delle consonanti polacche alveolari e prepalatali, per produrre le consonanti postalveolari italiane bisogna posizionare la lingua nello spazio esattamente tra i punti di articolazione dei foni polacchi corrispondenti. Detto ciò, si può concludere che $\mathrm{i}$ fonemi postalveolari italiani $/ \int 3 \mathrm{t} \int \mathrm{d} 3 /$ hanno due punti di riferimento

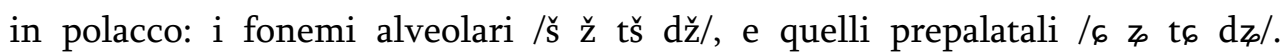
Interessante a questo porposito sarebbe approfondire il tema per verificare

Dizionario di Pronuncia Italiana usa ampiamente il simbolo /3/, che appare in parole già poco esotiche per gli italiani, come garage. 
quale gruppo dei foni polacchi venga percepito come più vicino agli italiani foni postalveolari.

Un altro gruppo di suoni che sono considerati pressappoco uguali, ma che allo stesso tempo mostrano una certa divergenza nel luogo di articolazione tra i fonemi italiani e quelli polacchi, sono i suoni nasali raffigurati nella tabella (2) dai simboli $/ \mathrm{n} / \mathrm{e} / \mathrm{n} /{ }^{17}$. Secondo la classificazione riportata nella tabella (2), nell'italiano $/ \mathrm{n} /$ è alveolare, mentre nel polacco è dentale. Secondo Canepari (2006), però, questa consonante anche in italiano può essere realizzata come un fono dentale, come una variante del fonema. Può darsi che proprio per questo motivo né polacchi né italiani di solito non percepiscano nessuna differenza tra la /n/ polacca e quella italiana. Sembra che nel caso della consonante /n/ i variegati punti di articolazione siano praticamente impercettibili.

La percezione del fono $/ \mathrm{n} /$ è tutta diversa ${ }^{18}$. Nonostante il fonema $/ \mathrm{n} /$ in italiano sia definito come palatale e in polacco come prepalatale ${ }^{19}$ - per cui la differenza tra le due realizzazioni sembra minima ed analoga a quella di /n/ Canepari (2006) osserva che in italiano non esiste nessuna variante di questo fonema. In altre parole, esso viene sempre realizzato come una consonante prepalatale, a differenza di $/ \mathrm{n} /$ che, per l'applicazione di qualche processo assimilatorio può diventare, per esempio, dentale.

Nonostante ciò, se esaminiamo la trascrizione fonetica di una parola con il fono /n/, per esempio bagno/'banno/, osserviamo che la consonante nasale / $\mathrm{n} /$ è rappresentata come geminata, una consonante doppia. Infatti, in italiano la consonante $/ \mathrm{n} /$ è tradizionalmente considerata una consonante rafforzata oppure una geminata intrinsica (Bertinetto e Loporcaro 2005), ed esso è dovuto al fatto che nella posizione intervocalica tale consonante viene sempre realizzata come una consonante lunga ${ }^{20}$. Dal punto di vista sia della fonologia

17 Un po'allarmante può risultare il fatto che per codificare tutte e due le varianti sia della $/ \mathrm{n} / \mathrm{sia}$ della $/ \mathrm{n} /$ in ogni caso l'alfabeto IPA utilizzi un solo simbolo (IPA, 2019). Comunque, le incongruenze di questo tipo all'interno della simboleggiatura dell'IPA non sono isolate, come lo mostra, per esempio, la discussione sul modo di trascrizione delle fricative e affricate (post)alveolari. Poiché l'argomento della classificazione dell'IPA non rientra nell'ambito del presente articolo, semplicemente diamo per scontato che il simbolo / $\mathrm{n} /$ venga usato per rappresentare due fonemi nasali diversi, ossia prepalatali e palatali, e che lo stesso valga per la consonante $/ \mathrm{n} /$.

${ }^{18}$ La Kwapisz-Osadnik (2012) ritiene che la consonante / $\mathrm{n} /$ abbia lo stesso luogo di articolazione in italiano e in polacco, essendo una consonante palatale.

${ }^{19}$ Secondo la Kwapisz-Osadnik (2012), il fono /n/ è palatale sia in italiano, sia in polacco. Non si spiega perché e su quali fonti si basa quella classificazione.

${ }^{20}$ Per l'analisi dettagliata della natura intrinseca di alcune geminate italiane, si veda l'articolo di Chierchia (1986). 
che della fonetica, questo è un fattore molto rilevante perché rende le due realizzazioni di questo suono sostanzialmente diverse, dato che in polacco la consonante $/ \mathrm{n} /$ è tipicamente singola, ma può anche essere realizzata come geminata.

Occorre sottolineare che la nasale / $\mathrm{n} /$ non è l'unica consonante italiana che di solito è realizzata come geminata. Questo vale anche per le consonanti come $/ \mathrm{j} \wedge \int \mathrm{ts} \mathrm{dz} /$, dette in italiano geminate intrinseche (Bertinetto e Loporcaro 2005), le quali, se non all'inizio di una parola, vengono pronunciate con la durata di due elementi consonantici.

In genere, le geminate come tali sono in principio pronunciate diversamente in italiano e in polacco. Nella lingua italiana le consonanti doppie articolate con un'occlusione nel tratto vocale sono prive della cosiddetta fase di esplosione del primo fono. In polacco l'esplosione dopo il primo elemento della geminata di solito è presente. Nel sistema IPA l'assenza della fase di esplosione è rappresentata dal segno grafico $/ \%$. Il problema è illustrato dagli esempi riportati a seguito.

(2) La discrepanza nella realizzazione delle consonanti con la fase di esplosione
a.
PL: panna
['panna] o ['pan`na], 'signorina'
IT: panna
['pan`na]
b. PL: frappe ['frapp $\varepsilon]$ o ['frap $p \varepsilon]$
IT: frappé [frap"pe]
c. PL: pizza ['pitstsa] o ['pits`tsa]
IT: pizza ['pits'ssa] $]^{21}$

Gli esempi citati sopra sono tutti omonimi. Le parole riportate nel punto (3a) rappresentano il gruppo di falsi amici, mentre quelle dei punti (3b) e (3c) raffigurano internazionalismi, detti anche cognati (ing. cognates). Tralasciando il significato di questi esempi, visto che dal punto di vista del presente lavoro è rilevante e attinente solo la somiglianza della forma delle parole lasciando, perciò, da parte le vocali che escono dall'ambito di quest'articolo. Tutte le parole citate in (3) contengono consonanti doppie la cui articolazione include un'occlusione. Sono le consonanti nasali presenti in (3a), le occlusive del punto

\footnotetext{
${ }^{21}$ Alternativamente, ogni geminata può essere trascritta con il segno diacritico [:]. Invece della sequenza delle due consonanti, per esempio /pp/, si può utilizzare la trascrizione alternativa [p:].
} 
(3b), e le affricate del punto (3c). Gli esempi mostrano che in polacco le geminate come $/ \mathrm{nn} /, / \mathrm{kk} / \mathrm{e} / \mathrm{tsts} /$ sono pronunciate o con una fase esplosiva, o con due. Entrambe le realizzazioni sono percepite dai madrelingua polacchi come corrette e naturali. In italiano, invece, solo una di quelle due pronunce è accettabile: le parole come quelle riportate in (3) sono sempre realizzate senza la fase di esplosione nel primo elemento della geminata.

\section{Conclusioni}

L'esame degli inventari consonantici dell'italiano e del polacco mostra che i due sistemi sono abbastanza simili, ma che tra di loro esistono discrepanze che non possono essere ignorate nell'analisi comparativa di queste due lingue. La prima differenza consiste nella mancanza dei fonemi corrispondenti tra le due lingue, come la consonante italiana $/ \mathrm{N} /$ e il fonema polacco $/ \mathrm{x} /$. Il secondo problema di contrasto da notare è la presenza dei fonemi che risultano molto simili, come il fono italiano / $/$ / e quello polacco /š/, ma in realtà sono diversi per tre motivi: il luogo di articolazione, l'arrotondamento della bocca, ma anche la lunghezza intrinseca della consonante italiana / $/$ / La discussione si chiude con una breve presentazione contrastiva dell'ultima discrepanza in merito alla pronuncia delle geminate italiane e polacche, la cui articolazione richiede la formazione di un ostacolo nel tratto vocale. Nel polacco, le consonanti lunghe di questo tipo sono pronunciate o come due consonanti staccate, ovvero con due fasi di esplosione, o senza l'esplosione della prima consonante. In italiano, invece, solo la seconda realizzazione è accettabile.

\section{Bibliografia}

Albano Leoni F. e P. Maturi (1995/2018). Manuale di fonetica. Roma: Carocci.

Anderson, S. R. (2010). How many languages are there in the world? Linguistic Society of America, Brochure Series: Frequently Asked Questions. Retrieved 13.01.2019, from https://www.linguisticsociety.org/content/how-many-languages-are-thereworld

Bertinetto, P. M., Loporcaro M. (2005). The sound pattern of Standard Italian, as compared with the varieties spoken in Florence, Milan and Rome. Journal of the International Phonetic Association, 35/2, 131-151. Cambridge: Cambridge University Press.

Broniś, O. (2017). Analiza semantyczna i fonetyczna wybranych fałszywych przyjaciół polsko-włoskich. In M. Majewska (ed.), Wokół homonimii językowej (pp. 175-192). Warszawa: Wydawnictwo Naukowe UKSW.

Canepari, L. (2006). Avviamento alla fonetica. Torino: Giulio Einaudi.

Canepari, L. (1999/2009). Dizionario di pronuncia italiana. Bologna: Zanichelli. 
Chierchia, G. (1986). Length, Syllabification and the Phonological Cycle in Italian. Journal of Italian Linguistics 8, 5-34. Pisa: Pacini Editore.

D'Achille, P. (2003/2010). L'italiano contemporaneo. Bologna: il Mulino.

Dardano, M., Trifone, P. (1995/2016). Grammatica italiana con nozioni di linguistica. Bologna: Zanichelli.

Hayes, B. (1989). Compensatory Lengthening in Moraic Phonology. Linguistic Inquiry 20, 253-306. Cambridge, Mass.: The MIT Press.

International Phonetic Alphabet (2019). Retrieved 27.01.2019, from http://www.internationalphoneticalphabet.org/ipa-charts/ipa-symbols-chartcomplete/

Karaś H. (2010). Dialekty i gwary polskie. Kompendium internetowe. Retrieved from http://www.dialektologia.uw.edu.pl/index.php?11=start

Kwapisz-Osadnik K. (2012). Podstawowe wiadomości z gramatyki polskiej $i$ włoskiej: szkic porównawczy. Katowice: Wydawnictwo Uniwersytetu Śląskiego.

Maturi P. (2006/2014). I suoni delle lingue, i suoni dell'italiano. Bologna: il Mulino.

McCarthy, J.J., Prince, A. (1993). Prosodic Morphology I: Constraint Interaction and Satisfaction. Rutgers University Center for Cognitive Science.

McCarthy, J.J., Prince, A. (1995). Faithfulness and Reduplicative Identity. Amherst and New Brunswick: University of Massachusetts and Rutgers University, University of Massachusetts Occasional Papers in Linguistics 18: Papers in Optimality Theory. 10.

Mioni, A. M. (1993). Fonetica e fonologia. Introduzione all'italiano contemporaneo: le strutture, a cura di A. A. Sobrero, 101-39. Roma: Laterza.

Ostaszewska, D., Tambor J. (2000). Fonetyka i fonologia współczesnego języka polskiego. Warszawa: Wydawnictwo Naukowe PWN.

Prince, A., Smolensky, P. (1993/2004). Optimality Theory: Constraint Interaction in Generative Grammar. Blackwell, Oxford, [Revision of 1993 technical report, Rutgers University Center for Cognitive Sciences. Available on Rutgers Optimality Archive, ROA-537].

Repetti, L. (1993). The Integration of Foreign Loans in the Phonology of Italian. Italica 70, 182-196.

Rubach, J. (1977). Changes of consonants in English and Polish. A generative account. Wrocław: Wydawnictwo Polska Akademia Nauk.

Rubach, J. (1984). Cyclic and lexical phonology: the structure of Polish. Dordrecht: Foris.

Rubach, J. (2000a). Glide and glottal stop insertion in Slavic languages: A DOT analysis. Linguistic Inquiry, 31.2, 271-317. Cambridge, Mass.: The MIT Press.

Rubach, J. (2000b). Backness switch in Russian. Phonology, 17, 39-64. Cambridge: Cambridge University Press.

Rubach, J. (2018). Surface Velar Palatalization in Polish. Natural Language and Linguistic Theory, 1-42. Springer Netherlands. https:/doi.org/10.1007/s11049018-9430-3 
Rydzewski, P. (2016). A Polish Argument for the Underlying Status of [i்]. Studies in Polish Linguistics, 11, 111-131.

SIL International (2019). Ethnologue. Retrieved 13.01.2019, from https://www.ethnologue.com/browse/names

Trubetzkoy, N. (1939). Grundzüge der Phonologie. 5. ed. Göttingen: Vandenhoeck \& Ruprecht.

Wierzchowska, B. (1971). Wymowa polska. Warszawa: Państwowe Zakłady Wydawnictw Polskich.

Wierzchowska, B. (1980). Fonetyka i fonologia języka polskiego. Wrocław: Zakład Narodowy im. Ossolińskich (PAN).

Wiśniewski, M. (2001). Zarys fonetyki i fonologii współczesnego języka polskiego. Toruń: Wydawnictwo UMK. 
